\title{
Production laitière dans la zone sylvopastorale du Sénégal : étude des facteurs de variation et modes de gestion par les populations locales
}

\author{
A.T. Diop ${ }^{1 *}$ A. Ickowicz ${ }^{2}$ M. Diène ${ }^{3}$ J.C. Nzimulinda ${ }^{4}$
}

Mots-clés

Production laitière - Biomasse Pâturage - Teneur en eau Télédétection - Sénégal.

\begin{abstract}
Résumé
Dans la région sahélienne du Sénégal, la production laitière des systèmes pastoraux connaît des variations interannuelles et intra-annuelles du fait que l'alimentation du cheptel est basée presque exclusivement sur les ressources naturelles. Les études menées à partir d'un dispositif de suivi dans les campements d'éleveurs et en station, et des données de la société Nestlé ont montré que la production laitière était fortement liée à la date de démarrage de la collecte, mais faiblement à la pluviométrie totale. La relation entre la production laitière totale et les indices de végétation normalisés (NDVI) était de type polynomial. Le pic de la production a été atteint au moment où la teneur en eau des fourrages a été de 70 p. 100. Des enquêtes auprès de pasteurs (hommes et femmes) appartenant à différentes ethnies et résidant dans les différents soussystèmes de production (Walo, Djoloff et Ferlo) ont indiqué que la durée de lactation, le nombre de vaches et les espèces animales ont constitué les principaux facteurs de variation de la production laitière. En fonction de l'ethnie à laquelle ils appartenaient, différents acteurs ont été responsables de la gestion du lait. Le caillage et l'extraction du beurre ont constitué les deux modes de conservation, et cinq types de calebasses ont été utilisés lors du processus. Les produits laitiers occupaient encore une place importante dans les revenus agricoles et le lait caillé écrémé était le produit le plus commercialisé.
\end{abstract}

Dans les régions sahéliennes d'Afrique où l'élevage est conduit principalement selon le mode extensif, la production laitière joue un rôle très important notamment chez les populations peules, comme celles de la zone sylvopastorale (ZSP) du Sénégal. Elle est la base de l'alimentation des populations qui en tirent aussi d'importants revenus $(2,4)$. Plusieurs études axées sur l'aptitude laitière des races bovines locales (zébu Gobra) et introduites

1. Isra-Lnerv / PPZC, BP 2057, Dakar Hann, Sénégal.

2. Cirad, UMR Elevage des ruminants en régions chaudes, Montpellier, F-34398

France.

3. SRA/CRZ, Dahra, Sénégal.

4. UCAD/ISE, Dakar, Sénégal.

* Auteur par correspondance

Tél. : +2218325615; fax : +2218323679

E-mail : amtadiop@orange.sn
(Guzéra, Pakistanais, Montbéliard, etc.) à produire du lait ont été réalisées en station de recherche $(6-9,16,17,26)$. D'autres études menées dans les campements $(4,15)$ ont permis de décrire les fluctuations de cette production selon les saisons et leur exploitation par les populations.

Avec le développement des centres urbains, le lait est acheminé depuis les zones pastorales sous différentes formes (frais, caillé, beurre de vache) pour être commercialisé. Malgré la compétition de la poudre de lait importée, sa contribution à l'approvisionnement des villes est de plus en plus importante, notamment à travers la mise en place de ceinture laitière périurbaine et l'amélioration des possibilités de transport. Avec ce nouveau dynamisme qui caractérise la production laitière, il est nécessaire de mieux comprendre les relations qui la lient aux facteurs environnementaux. Une analyse plus fine des modes de gestion des produits laitiers issus des systèmes pastoraux devrait aussi permettre d'assurer une meilleure contribution à la demande nationale en lait. 
Les objectifs de la présente étude ont été, d'une part, d'avoir une meilleure connaissance de la dynamique de production laitière en fonction de l'état des ressources fourragères et en eau et, d'autre part, d'analyser les systèmes de gestion des produits laitiers à la suite des tentatives d'amélioration techniques et économiques initiés par différentes organisations.

\section{MATERIEL ET METHODES}

\section{Cadre d'étude}

La zone sylvopastorale constitue une des régions agroécologiques du Sénégal (figure 1). Elle est située dans la partie sahélienne selon la classification d'Aubreville (1). La moyenne pluviométrique annuelle sur l'ensemble de la ZSP a baissé de $414 \mathrm{~mm}$, entre 1951 et 1970, à $270 \mathrm{~mm}$, entre 1971 et 1990, pour remonter à 288,3 mm, entre 1991 et 2001. Dans la période entre juin et octobre 98,5 p. 100 des pluies sont enregistrées et le mois d'août est le plus humide de l'année, deux années sur trois.

La végétation est une steppe arbustive composée d'un tapis herbacé à dominante d'espèces annuelles et d'une strate arbustive peu dense $(27,28)$. La biomasse herbacée est à son maximum à la fin de la saison des pluies (de la mi-septembre à début octobre). Elle varie selon les années de 100-200 à $2000-3000 \mathrm{~kg}$ de matière sèche/hectare (19). Le dessèchement progressif sur pied entraîne une perte de la valeur nutritive avec une disparition progressive de l'azote digestible en saison sèche (14). Les mares constituent la principale source d'abreuvement du cheptel et des humains en saison des pluies (4). En saison sèche, ce sont les puits mais surtout les forages qui sont fréquentés.

Les Peuls y sont dominants, suivis des Oulofs, puis des Maures. Mais de plus en plus de Sérères agropasteurs quittent le bassin arachidier pour s'y installer. L'élevage constitue l'activité première. Par exemple, dans la zone de Thiel, il représente 75,9 p. 100 des revenus des ménages ; il est suivi des activités agricoles $(20,6$ p. 100) et forestières $(0,4$ p. 100) (10). Il fournit une part importante des productions animales du Sénégal avec 35 p. 100 de l'effectif national de bovins et 50 p. 100 des petits ruminants (21). Les bovins sont essentiellement des zébus peuls (race Gobra). D'autres races locales comme les zébus Maures et des races exotiques comme les Pakistanais et les Guzéra y sont aussi rencontrées.

Les troupeaux bovins comprennent entre 72 et 75 p. 100 de femelles $(4,12)$. L'âge à la première mise bas est en moyenne de 4,4 ans. L'intervalle entre vêlages est en moyenne de 22,4 mois. La plupart des naissances ont lieu de la fin de la saison sèche au début de la saison des pluies (mai, juin et juillet). La durée de gestation de la vache Gobra est de 293 jours. La moyenne du poids des animaux croît régulièrement avec l'âge de 5 à 12 ans, mais elle est moins représentative à partir de 13 ans (12). La production laitière totale moyenne de la femelle Gobra est de 400 à $500 \mathrm{~kg}$ au cours d'une lactation qui ne dépasse pas 5 à 6 mois. Chez les Pakistanaises, elle est de $1147 \pm 126 \mathrm{~kg}$ pour une durée de lactation moyenne de 8 mois (9).

La complémentation des animaux avec des aliments concentrés était pratiquement inconnue jusqu'à la grande sécheresse de 19721973 (22). Elle s'est développée avec les activités de la Société de développement de l'élevage en ZSP (Sodesp). Les produits laitiers constituent un élément essentiel de l'alimentation des populations (3). Le lait est consommé frais avec le couscous (à base de mil et, de plus en plus, de riz), mais aussi sous forme de caillé (kossam kadam $=$ produit de la fermentation du lait du fait de la présence naturelle de bactéries lactiques dans le milieu) (20). La matière grasse est souvent récupérée pour faire du beurre. A partir de 1991, la société Nestlé Sénégal a lancé un programme de collecte de lait dans sept localités (Boulal, Warkhokh, Déali, Thiargny, Guéli, Thiamène, Wendou Loumbel). Les volumes collectés ont été cependant très faibles par rapport à ses objectifs. Le prix peu attractif $(90 \mathrm{Fcfa} / \mathrm{L}$ au démarrage, $136 \mathrm{Fcfa} / \mathrm{L}$ en 2002, contre 200 Fcfa sur le marché) explique en partie cette défaillance des éleveurs. Compte tenu de la saisonnalité de la production laitière, la collecte était maintenue seulement pendant la saison des pluies, les lieux de collecte et de transformation (Dakar) étant distants de
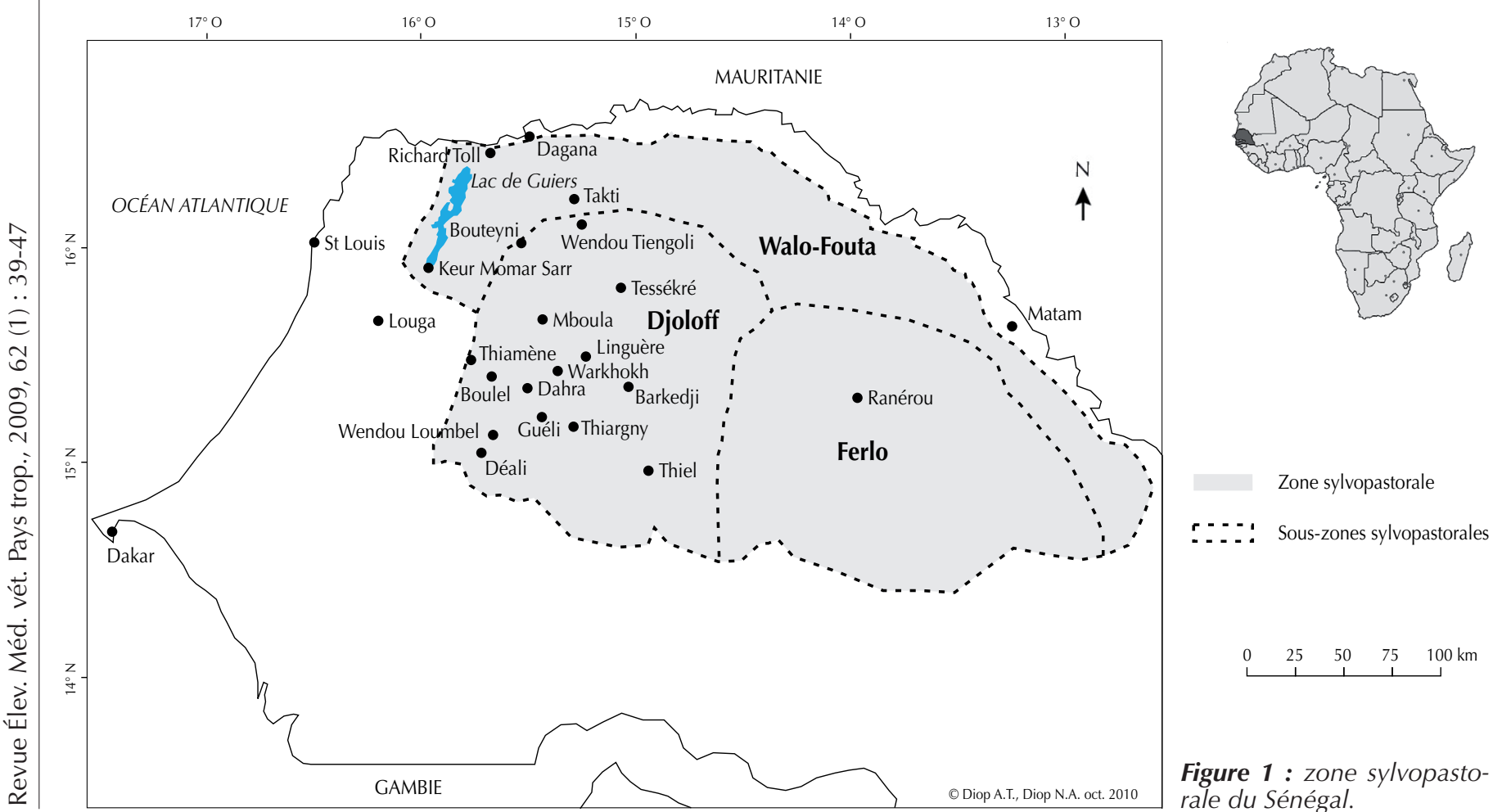

Figure 1 : zone sylvopastorale du Sénégal. 
300 kilomètres et la réfrigération obligatoire. Du fait d'un coût de collecte et de traitement non compétitif par rapport à l'utilisation de la poudre, Nestlé a arrêté l'opération en 2002.

\section{Collecte de données sur la production laitière}

\section{Utilisation des données de collecte de lait du service agricole} de Nestlé à Dahra

Une campagne de collecte de lait frais était ouverte chaque année au début de la saison des pluies et elle se terminait lorsque les quantités collectées devenaient faibles. L'hypothèse de travail retenue a consisté à considérer les quantités de lait collectées par Nestlé comme reflétant l'évolution de la production laitière dans la zone d'étude. Elle est fonction de multiples facteurs comme le nombre de vaches traites, la durée de la traite, l'intensité de la traite par l'éleveur, l'effort de collecte de lait par la société, la disponibilité alimentaire en eau et en fourrage, la fraction de lait commercialisée, etc. Dans le cadre du présent travail, il a été envisagé l'effet global de ces facteurs sur le potentiel laitier de la ZSP, les variables comme les effectifs de vaches traites et la quantité de lait produite par vache étant fortement liées au milieu. Les statistiques sur les quantités de lait de 1991 à 1999 ont été mises à la disposition des auteurs par la société à Dahra.

\section{Mise en place d'un dispositif de suivi de la production laitière}

Le Centre de recherche zootechnique (CRZ) de Dahra est situé au milieu de la ZSP. Pour les besoins de ses activités, il dispose d'un troupeau composé de Gobra (85 p. 100), de Guzéra (12 p. 100) et de métis Gobra - Guzéra (3 p. 100). Au début de chaque saison des pluies, une partie des vaches en lactation est regroupée. Comme pour le reste des bovins du CRZ, le troupeau fréquente les pâturages naturels de la zone clôturée du Centre. Pour les besoins de cette étude, 45 vaches de ce lot ont été suivies de juillet à novembre 1997. Elles n'ont pas reçu de complémentation pendant la période de suivi.

Quatre éleveurs résidant dans un campement d'éleveurs peuls à trois kilomètres de Dahra ont été choisis. Ce choix a été guidé à la fois par la facilité d'accès au campement et du fait que ces éleveurs avaient l'habitude de collaborer avec le CRZ. Le suivi de la quantité de lait a été fait tous les 15 jours durant les saisons des pluies de 1997 et de 2000. Il a porté à chaque passage sur la mesure de la production globale du matin d'un total de 41 vaches.

\section{Collecte de données sur les facteurs environnementaux}

\section{Données pluviométriques}

Selon la littérature $(8,16,26)$, le facteur pluviométrique est le facteur le plus important dans l'évolution des performances de production du cheptel local dans les zones tropicales. Les données pluviométriques journalières du CRZ de Dahra ont ainsi été utilisées pour la période allant de 1992 à 1999.

\section{Données de biomasse herbacée}

Il s'agit, d'une part, des données du Centre de suivi écologique (CSE) et, d'autre part, de celles issues du dispositif de suivi mis en place pour les besoins de l'étude. Depuis 1984, la ZSP fait l'objet d'un suivi annuel par le CSE de l'évolution de l'indice de végétation (normalized difference vegetation index : NDVI), à partir des données du satellite météorologique NOAA pour l'élaboration de cartes de biomasse herbacée en fin de saison des pluies. Les auteurs ont basé leur travail sur ces données mises à leur disposition par le CSE (cumul des maximums de NDVI décadaire entre juin et octobre sur un rayon de 20 kilomètres autour de Dahra pour la période allant de 1991 à 1996).
D'autre part, les auteurs ont mis en place un dispositif de suivi de la biomasse herbacée au niveau des parcelles du CRZ et des parcours fréquentés par les troupeaux des campements faisant l'objet de suivi de production laitière : après chaque collecte de lait au niveau des troupeaux, des relevés de biomasse herbacée ont été réalisés (fauche totale de biomasse verte dans un carré d'un mètre de côté répétée 10 à 20 fois selon l'hétérogénéité du couvert). Par la suite, des échantillons ont été prélevés pour la détermination de la teneur en eau des herbacées.

\section{Enquêtes auprès des populations pastorales sur leurs pratiques de production laitière}

Au niveau de la ville de Dahra et autour des centres de collecte de la société Nestlé, une vingtaine de personnes pratiquant l'élevage laitier ont été rencontrées entre 1996 et 1998. Les entretiens ont porté sur leurs pratiques de production et de commercialisation des produits laitiers.

Par ailleurs, dans les différentes sous-unités de la ZSP (Djoloff, Ferlo et Walo-Fouta), des entretiens ont eu lieu durant l'année 2001, avec des personnes ressources, notamment des femmes de Boulal, Déali, Dahra, Tatki, et Ranérou.

\section{RESULTATS ET DISCUSSION}

\section{Effet de la pluviométrie sur la production laitière}

L'évolution de la production laitière par rapport à la pluviométrie et l'année de collecte (tableau I) a permis de faire les constats ciaprès.

Les premières pluies ont été enregistrées à la semaine 25 et la collecte de lait a débuté 7,6 semaines $( \pm 0,8 ; \mathrm{n}=7)$ plus tard, c'est-à-dire à la semaine $33( \pm 1,4 ; n=7)$. Aucun effet n'a été présent entre le début de la saison des pluies et le début de la collecte du lait ou la production laitière collectée. Cependant, le niveau de précipitation au démarrage de la collecte de lait a été relativement constant avec une quantité de pluie égale à $143,8 \mathrm{~mm}$ $( \pm 26,5 ; n=7)$. Les auteurs ont constaté que plus la collecte de lait commençait tôt, plus la production totale de lait était forte. En effet, la date de début de la collecte de lait (x) a été fortement en relation avec la production laitière totale $(\mathrm{y}=-889,54 \mathrm{x}+31591$; $\left.\mathrm{r}^{2}=0,9193\right)$;

L'analyse de l'évolution de la collecte de lait en fonction de la pluviométrie pour l'année 1992 (très déficitaire) a montré que le pic de collecte était atteint trois semaines après l'arrêt des pluies, alors que pour les autres années, le pic était survenu en pleine période de pluies. Une relation linéaire existait cependant entre le pic de collecte de lait (y) et la date de début de collecte de lait (x) :

$\left[y=0,8056 x+1,5278\left(r^{2}=0,6489\right)\right]$.

De 1993 à 1996, le pic de collecte a eu lieu en moyenne à 5,8 semaines $( \pm 1,9 ; n=7)$ après le début de collecte, soit à la semaine $39(38,8 \pm 1,7)$ en moyenne, c'est-à-dire à la deuxième quinzaine de septembre. Le niveau des précipitations au pic de collecte a été de $293,2 \mathrm{~mm}( \pm 46 ; \mathrm{n}=7)$.

La chute de la production laitière est survenue dans la semaine qui a suivi celle du pic pour atteindre 50 p. 100 du niveau de collecte du pic après quatre semaines $( \pm 1 ; n=7)$. Sauf en 1994 où le niveau de collecte est resté à environ 25 p. 100 du pic jusqu'à l'arrêt de la collecte, la baisse du niveau de collecte a atteint 75 p. 100 de la collecte au pic en moyenne à six semaines $( \pm 0,7 ; \mathrm{n}=7)$. Aucune relation entre l'intensité de la baisse du niveau de collecte de lait et les caractéristiques de la pluie n'a cependant été notée. 


\section{Tableau I}

Evolution de la production laitière en fonction de la pluviométrie

\begin{tabular}{|c|c|c|c|c|c|c|c|c|}
\hline & 1992 & 1993 & 1994 & 1995 & 1996 & 1997 & 1998 & 1999 \\
\hline Début des pluies ( $\mathrm{n}^{\circ}$ de la semaine) & 27 & 26 & 24 & 25 & 24 & - & 30 & 28 \\
\hline Début de collecte de lait ( $\mathrm{n}^{\circ}$ de la semaine) & 35 & 34 & 32 & 31 & 32 & - & 34 & 31 \\
\hline Niveau de pluie au début de la collecte $(\mathrm{mm})$ & 154,4 & 188,7 & 135 & 117,4 & 120,9 & - & 138,5 & 95,8 \\
\hline Pic de collecte de lait ( ${ }^{\circ}$ de la semaine) & 42 & 39 & 38 & 38 & 37 & - & 39 & 38 \\
\hline Niveau de pluies au pic de collecte (mm) & 228,8 & 307,1 & 258,8 & 359,2 & 314,8 & - & 225,3 & 459,5 \\
\hline $\begin{array}{l}\text { Baisse de } 50 \% \text { du niveau de collecte } \\
\text { par rapport au pic (semaines) }\end{array}$ & 4 & 3 & 6 & 3 & 4 & - & 5 & 5 \\
\hline $\begin{array}{l}\text { Baisse de } 75 \% \text { du niveau de collecte } \\
\text { par rapport au pic (semaines) }\end{array}$ & 5 & 7 & 13 & 6 & 6 & - & 6 & 7 \\
\hline Arrêt des pluies ( $\mathrm{n}^{\circ}$ de la semaine) & 39 & 42 & 43 & 41 & 42 & - & 41 & 42 \\
\hline Arrêt de collecte de lait ( $\mathrm{n}^{\circ}$ de la semaine) & 52 & 52 & 52 & 52 & 49 & - & 49 & 51 \\
\hline Production lait totale $(\mathrm{hL})$ & 600 & 800 & 3050 & 3429 & 3444 & - & 1624 & 4489 \\
\hline Rang d'année de collecte & 1 & 2 & 4 & 5 & 6 & - & 7 & 8 \\
\hline
\end{tabular}

«-» Données manquantes

La période d'arrêt des pluies n'a eu d'effet ni sur la période d'arrêt de collecte de lait, ni sur la production laitière totale (figure 2). En moyenne, elle est survenue à la semaine $41( \pm 1,3 ; n=7)$. Cependant la collecte de lait a continué jusqu'à la fin de l'année (semaine $52)$. Elle s'est étalée sur 13,2 semaines $( \pm 0,8 ; n=7)$ après le pic de collecte et 10 semaines $( \pm 0,7 ; n=7)$ après l'arrêt des pluies. A la fin de la saison des pluies, le niveau de collecte de lait a été de 53,1 p. 100 ( $\pm 6,2$ p. 100) par rapport au pic de collecte, et au moment de l'arrêt de la collecte il a été de 9,8 p. $100( \pm 7,9$; $\mathrm{n}=7$ ).

Le rang de l'année de collecte de lait (x) a semblé cependant en relation avec la période d'arrêt de collecte de lait $(\mathrm{y}=-0,3551 \mathrm{x}+$ 52,$\left.674 ; \mathrm{r}^{2}=0,4514\right)$.

Enfin, la pluie totale $(\mathrm{x})$ a présenté une faible relation avec la production laitière totale $\left(\mathrm{y}=9,2974 \mathrm{x}-678,24 ; \mathrm{r}^{2}=0,4256\right)$.

L'évolution de la pluviométrie par rapport à la collecte de lait, d'une part, au CRZ de Dahra et dans les campements d'éleveurs en 1997 (figure 3) et, d'autre part, au niveau des campements d'éleveurs en 2000 (figure 4) a montré que la saison des pluies a démarré plus tôt en 1997. La traite des animaux a cependant commencé plus tôt en

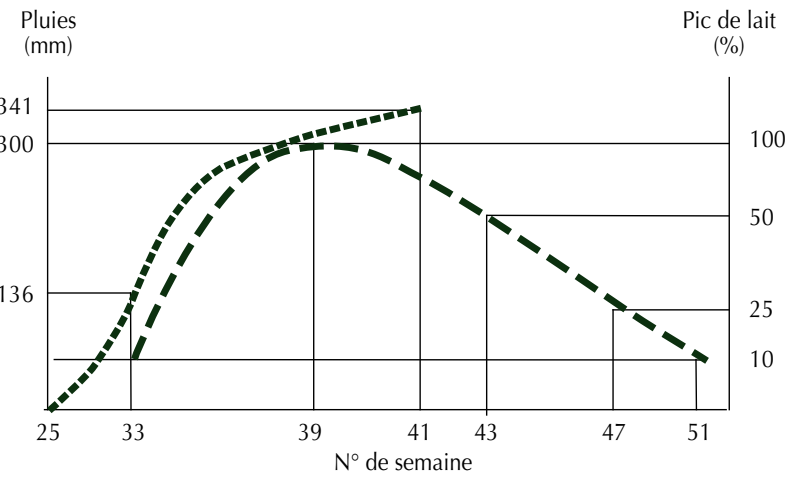

Figure 2 : relation entre l'évolution des pluies et la production laitière.
2000 (28 juillet au lieu d'août en 1997). Dans tous les cas, la hauteur d'eau enregistrée était aux alentours de $50 \mathrm{~mm}$.

Les quantités de pluie ont été plus importantes en 2000 (359 mm) qu'en 1997 (287 mm). Ceci a probablement permis d'avoir dans les campements une durée de traite nettement plus longue en 2000 (cinq mois) qu'en 1997 (un peu plus de deux mois et demi). Par ailleurs, en 1997, les pluies se sont arrêtées à la semaine 41 (1 ${ }^{\text {re }}$ décade d'octobre). Des difficultés logistiques ont fait que le suivi de la semaine 40 n'a pas pu se faire. A la semaine 42, les éleveurs ont indiqué que le pic de production était déjà passé. La semaine 42 se trouvant dans la phase de décroissance, cela a permis de situer le pic de production entre les semaines 39 et 41.

La production de lait la plus élevée enregistrée en 1997 a été de 1,6 $\mathrm{L} /$ vache/jour. Du fait que cette valeur ne correspond pas au pic de cette année, on peut dire que par rapport à l'année 2000 où le pic a été de 1,7 L, la production laitière maximale par jour et par vache a été presque la même pour les deux années.

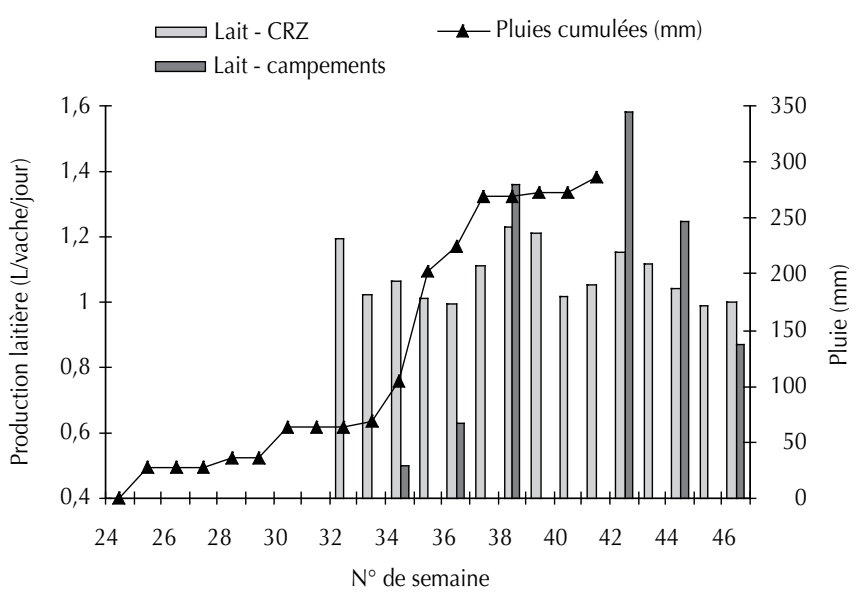

Figure 3 : évolution de la production laitière au niveau du Centre de recherche zootechnique (CRZ) de Dahra et des campements en relation avec la pluviométrie en 1997. 
Les courbes d'évolution de la quantité de lait de traite ont eu une allure sinusoïdale au CRZ de Dahra et en cloche dans les campements lors des deux années. Un pic de collecte de lait dans les campements a eu lieu vers la première semaine d'octobre en 1997 et un peu plus tôt (vers la mi-septembre) en 2000. Un aplatissement du pic de lactation peut être observé par suite d'une meilleure alimentation et dans de rares cas par transmission génétique (16).

La différence notée entre le CRZ et les campements pouvait être attribuée à deux facteurs. Le cheptel du CRZ bénéficiait d'une alimentation en eau régulière et suffisante durant toute l'année. Ce qui n'était pas le cas du cheptel des campements qui, avec le tarissement des mares en fin de saison des pluies, devait passer d'un abreuvement quotidien à un abreuvement tous les deux jours aux forages. La disponibilité fourragère du CRZ, même si elle baissait qualitativement, demeurait suffisante quantitativement plusieurs mois après la fin de la saison des pluies.

\section{Effet de la quantité de biomasse et de la teneur en eau des herbacées sur la production laitière}

Le NDVI a très souvent atteint son niveau maximum au mois de septembre (figure 5). Cette période correspondait également au maximum de collecte pour la production laitière entre 1993 et 1996. La relation entre la production laitière (y) et le NDVI (x) pendant cette période a été de type polynomial $\left(y=-7 \mathrm{e}^{-10} \mathrm{x}+3\right.$ $\left.\mathrm{e}^{-5} \mathrm{x}+0,0793 ; \mathrm{r}^{2}=0,6266\right)$.

Durant les années 1991 et 1992, des écarts entre les dates de maximum du NDVI et celles de la production laitière ont été notées ; le maximum de collecte de lait a eu lieu avec un mois de retard environ. En outre, en 1992 et 1993, les courbes de productivité laitière ont été moins élevées que celles du NDVI. Les caractéristiques des pâturages (composition floristique, stade végétatif) étaient probablement à l'origine de ces déphasages.

En ZSP, la biomasse herbacée est évaluée depuis 1980 en utilisant le NDVI à partir des données du satellite NOAA $(12,16)$. Les cartes ainsi obtenues permettent aux services nationaux de développement de mieux planifier leurs activités, même si des études ont montré que la relation entre le NDVI et la biomasse était faible en ZSP (11, 23). Dans la perspective de la collecte de lait pour les industries de transformation ou pour la vente dans les centres urbains, la détermination du niveau de biomasse herbacé par le NDVI, en raison de la corrélation avec le niveau de production laitière maximum, offrirait une information importante pour

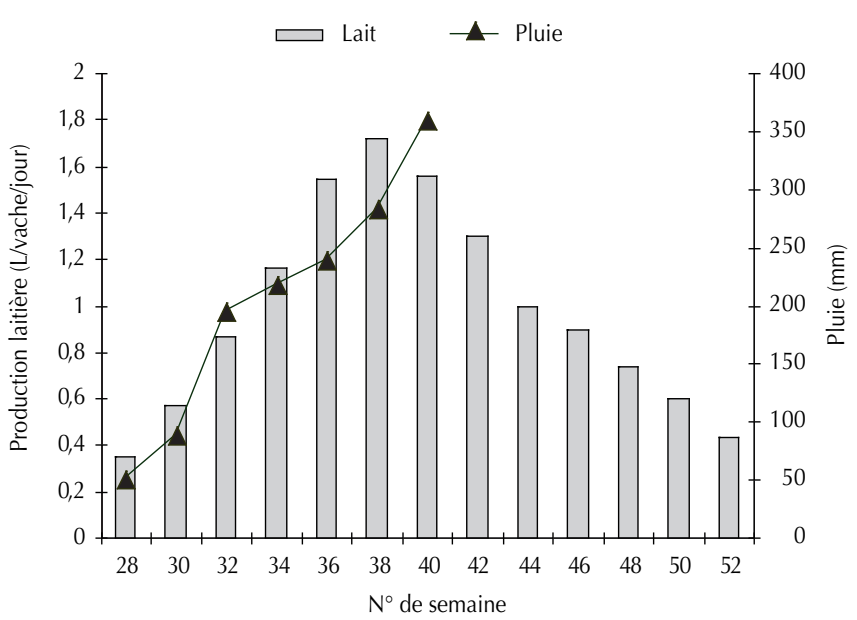

Figure 4 : évolution de la production laitière au niveau des campements en relation avec la pluviométrie en 2000. l'organisation de cette collecte. Des études complémentaires devraient alors être entreprises pour confirmer cette corrélation et améliorer cet indice pour parvenir à une meilleure prévision.

La courbe relative à la teneur en eau des herbacées des pâturages naturels et la quantité de lait traite par vache et par jour a montré que les quantités de lait collectées en 1997 et 2000 (figure 6) augmentaient progressivement pour atteindre un pic au moment où les fourrages avaient une teneur en eau d'environ 70 p. 100. Au démarrage de la traite, la teneur en eau des fourrages dépassait 90 p. 100. La baisse du volume de lait trait s'est poursuivie parallèlement à celle de la teneur en eau des fourrages.

En 1997, la traite a été interrompue à 0,87 L/vache/jour au moment où la teneur en eau des fourrages était de 31,5 p. 100 et en 2000 à $0,43 \mathrm{~L} / v a c h e / j o u r$ avec une teneur en eau des fourrages de 20 p. 100. Pour les deux années, la teneur en eau des fourrages de 30 p. 100 correspondait à peu près à la même quantité de lait traite, entre 0,87 et $0,90 \mathrm{~L} /$ vache/jour.

L'importance de l'eau pour la lactation des vaches a été signalée par de nombreux auteurs. Le besoin supplémentaire en eau d'abreuvement est estimé entre 1,5 à $3 \mathrm{~L}$ pour un litre de lait produit par vache $(15,26)$. La consommation est influencée entre autres par la température ambiante, la teneur en matières sèches de la ration et le niveau de production de lait (30).

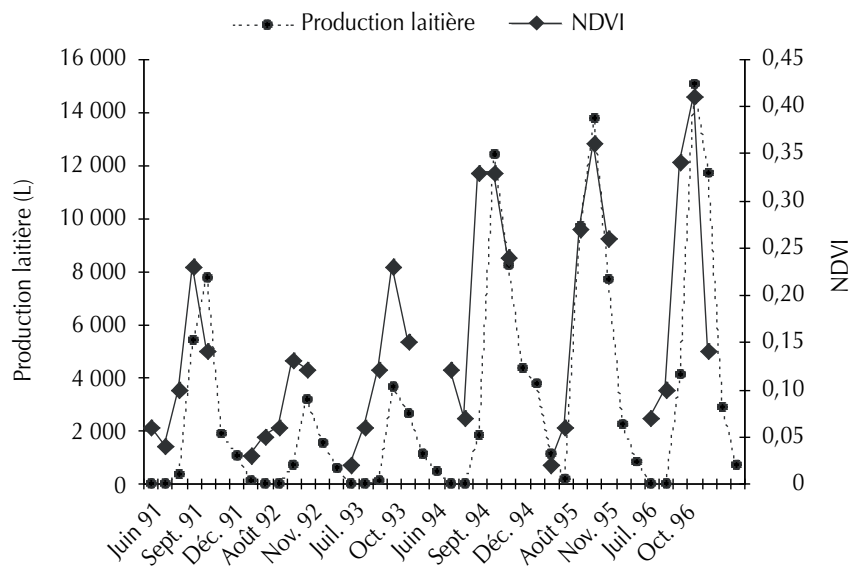

Figure 5 : évolution mensuelle de la production laitière et de l'indice de végétation (NDVI) de 1991 à 1996.

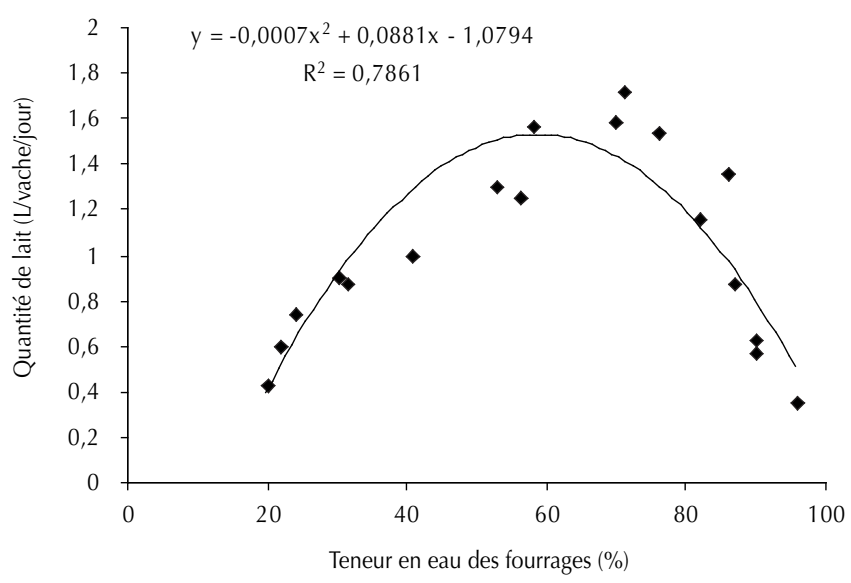

Figure 6 : évolution de la quantité de lait en fonction de la teneur en eau des fourrages en 1997 et 2000 en zone sylvopastorale au Sénégal. 
Ainsi, la production laitière des vaches en ZSP reste très liée aux caractéristiques des pâturages. Ces fourrages apportent des éléments nutritifs mais aussi de l'eau. Si leur teneur en eau diminue, il est nécessaire de pallier cette perte. Cependant, en ZSP, en même temps que l'eau des fourrages diminue, les mares s'assèchent, créant un déficit énorme dans les possibilités d'abreuvement des vaches et donc dans le maintien de la production laitière. De fait, l'éleveur arrête la traite dès que les mares s'assèchent, traduisant la situation par ces mots : «l'eau des forages ne permet pas d'avoir du lait ».

\section{Facteurs zootechniques de variation de la production laitière}

La figure 7 indique les facteurs de variation de la production laitière selon les éleveurs.

\section{Espèces animales traites}

Selon les éleveurs, la production laitière du troupeau en ZSP était issue de la traite des femelles allaitantes des espèces bovines, ovines et caprines. Les camélidés étaient peu présents en comparaison aux autres espèces et leur lait utilisé surtout par les Maures. Selon les espèces animales traites, des différences de pratiques ont aussi été signalées en fonction de l'ethnie des éleveurs : les Peuls, comme les Jenngelbe autour de Dahra et Thiel, et les Mbaaranaabe près de Déali trayaient uniquement les vaches mais pas les petits ruminants. D'autres, comme les Bisnaabe et les Pampinabe autour des forages de Tatki et de Widou, trayaient en plus les petits ruminants. Toutefois, certains parmi eux ne trayaient pas les brebis.

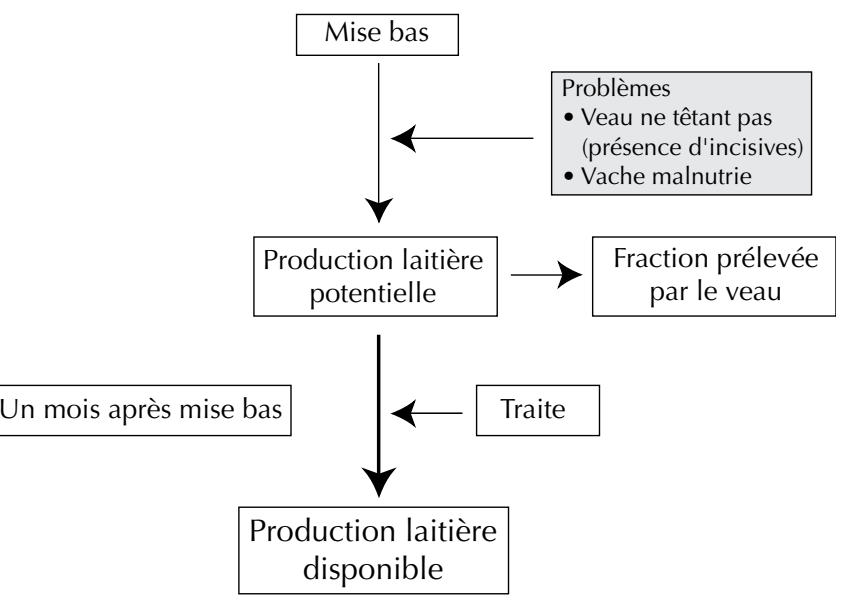

- Espèce

Bovin

Ovin, caprin : selon famille; selon fraction d'appartenance ; en cas d'impossibilité d'accès au lait de bovin

- Effectif : en fonction du nombre d'animaux ayant mis bas

- Périodicité : 1 fois / jour (matin) ; 2 fois / jour (matin et soir)

- Période : saison des pluies (vaches et petits ruminants); saison sèche (petits ruminants)

- Durée lactation (vache) : de 1 à 2 ans

- Complémentation : selon possibilité de commercialisation du lait

- Possibilité de commercialisation (proximité d'un lieu de commercialisation) ("forage », centre urbain ; existence d'une structure de commercialisation )

Figure 7 : facteurs de variation de la production laitière dans la zone sylvopastorale du Sénégal.
La traite des brebis est d'abord une question de disponibilité en lait. Les éleveurs qui n'ont pas un nombre important de vaches sont obligés de traire les brebis. Certains éleveurs considèrent que la traite des brebis permet souvent d'éviter aux agneaux d'avoir une indigestion liée à l'ingestion d'une trop grande quantité de lait. En période de transhumance, les bergers qui conduisent les petits ruminants sont aussi obligés de consommer leur lait. Parfois, les vaches n'ont pas suffisamment de lait, surtout en période de déficit fourrager. Il importe alors d'avoir recours aux petits ruminants pour la consommation familiale. La traite des petits ruminants est de plus en plus courante pour améliorer le revenu des éleveurs, notamment ceux qui sont proches des centres urbains.

\section{Périodicité et période de traite}

Selon les résultats d'entretiens, la traite avait lieu principalement, durant la saison des pluies (ndoungou), une ou deux fois par jour selon les galleeji. Pour la plupart des éleveurs, au fur et à mesure que la saison sèche avançait, le lait de la vache était laissé presque entièrement au veau.

\section{Durée de lactation}

La traite démarrait un mois après la mise bas pour permettre aux veaux de se développer. Des perturbations dans la lactation ont été parfois notées, comme le veau refusant de téter par suite d'une stomatite à la naissance ou de la présence prématurée de dents. Les vaches étaient en lactation durant une à deux années.

Le sevrage a lieu dès que la vache est gravide. Ce sont les hommes ou les femmes qui s'en occupent. Plusieurs stratégies, semblables à celles signalées par Baron (3) en milieu éleveur mauritanien (Maures et Peuls), sont utilisées par les éleveurs pour empêcher la tétée d'un jeune animal. L'une d'elles consiste à séparer le jeune de sa mère en l'amenant par exemple dans un autre troupeau. Une autre est de créer un obstacle pour éviter l'accès au pis, soit avec un morceau de calebasse taillé au niveau des naseaux (tognal), soit avec un bois taillé (tutal) dans les naseaux (figure 8), soit en déposant des fèces de petits ruminants sur les mamelles de la vache.

\section{Nombre de vaches à traire}

Comme indiqué précédemment, la quantité de lait fournie par une vache dépasse en saison des pluies un litre par jour. La consommation de lait (frais et caillé) d'un adulte par jour est estimée à 1,5 L/ individu pendant la saison de production (saison des pluies) (4, 5). Pour une famille de dix personnes, il serait donc nécessaire de traire à peu prés 15 vaches, ce qui justifie la nécessité d'avoir beaucoup de vaches dans un troupeau.

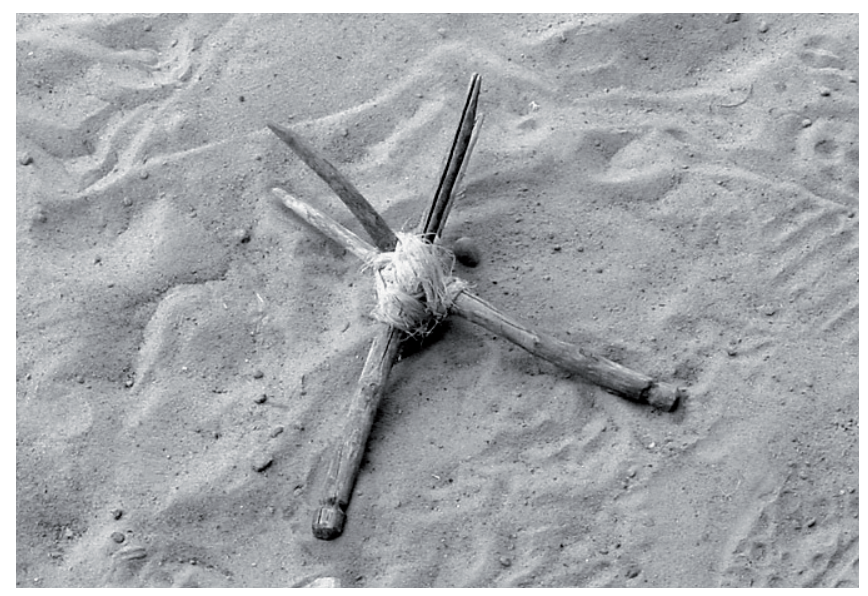

Figure 8 : tutal - bois taillé destiné à empêcher l'accès $d u$ veau au pis de la vache. 


\section{Complémentation}

Avec le développement de la commercialisation des produits laitiers, des quantités de lait de plus en plus importantes sont tirées du troupeau pendant la saison des pluies. Ceci amène certains galleeji à apporter des compléments aux vaches pour pouvoir vendre du lait pendant la saison sèche. A part certaines zones de forages (Boulal et Wendou Loumbel, par exemple) et des éleveurs qui se sont établis en zone périurbaine, cette pratique n'est pas très répandue. La traite est arrêtée dès que les pâturages ne sont plus suffisants pour l'alimentation du cheptel. Cependant, une pratique de plus en plus courante dans certaines familles d'éleveurs installés dans les villes comme Dahra consiste à amener, en saison sèche, des vaches en lactation au niveau de leur maison en ville pour les complémenter. Le lait trait est vendu à $300 \mathrm{Fcfa} / \mathrm{L}$ au lieu de $150 \mathrm{Fcfa} / \mathrm{L}$ et les revenus permettent d'acheter le complément. Dès l'apparition du tarissement, la vache est ramenée dans le troupeau en extensif.

\section{Acteurs responsables de la gestion des produits laitiers}

Des différences en fonction de l'ethnie sont notées dans l'exploitation des produits laitiers. Ainsi, chez les Peuls, chaque épouse a un certain nombre d'animaux à traire et ce sont ces animaux-là dont elle s'occupe. C'est elle qui gère le lait collecté et assure la transformation et la commercialisation du surplus.

Chez les Oulofs, c'est la mère (épouse du chef de la concession) qui est responsable de la production laitière. Ainsi, après la traite, toutes les épouses vivant dans la même concession apportent leur lait et c'est elle qui procède à la répartition entre les différentes destinations. S'il reste du lait frais, il est caillé et la matière grasse est enlevée pour faire du beurre. Le caillot n'est pas vendu mais le plus souvent distribué.

Chez les agropasteurs sérères du bassin arachidier qui viennent en transhumance en ZSP pendant la saison des pluies ou ceux qui résident dans la zone, ce sont les hommes (bergers) qui font la traite, mais l'extraction du beurre est une activité des femmes.

\section{Répartition de la production laitière de la vache}

Les données de Schaffer ( 25 ; tableau II) indiquent que la quantité de lait consommée par les veaux d'un troupeau peut être estimée à 34 p. 100 de la production laitière totale du troupeau dans une zone comme Widou Thiengoli et Bouteyni, habitée principalement par des Peuls.

La quantité de lait utilisée par la famille est fonction du groupe ethnique, des possibilités de commercialisation et des quantités de lait offertes par le troupeau. Ainsi, au fur et à mesure que la saison sèche avance, la production laitière diminue. Toute la production

\section{Tableau II}

Destination de la production laitière totale au niveau de Widou Thiengoli et Bouteyni en zone sylvopastorale

\begin{tabular}{lc} 
Destination & \% de la production \\
\hline Autoconsommation & 35,4 \\
Laissé au veau & 34 \\
Vente de lait frais/jour & 6,4 \\
Vente de lait caillé/jour (écrémé) & 24,2 \\
Total & 100
\end{tabular}

Source : Schaffer, 1994 laitière de la vache est laissée au veau. C'est d'ailleurs à partir de cette période que l'on fait le plus appel à la production laitière des petits ruminants pour la consommation familiale et la vente.

\section{Caillage et extraction du beurre : deux modes principaux de transformation et de conservation du lait}

Les récipients utilisés pour la collecte et la transformation du lait sont faits avec des matériaux végétaux. Chez les Peuls, ce sont des calebasses en bois. Certains arbres comme Balanites aegyptiaca transmettent leur goût au lait. Sclerocarya birrea est l'arbre le plus utilisé, mais à cause de fortes mortalités encourues chez cette espèce ces dernières années, il est de plus en plus rare. Chez les Sérères, ils sont faits avec le fruit de Lagenaria siceraria (une cucurbitacée). La calebasse utilisée pour la traite n'est pas la même que celle utilisée pour le caillage. Il y a ainsi autant de calebasses que de destinations du lait.

Un bon caillage s'obtient avec une calebasse faite avec un bois mûr et ferme, et un couvercle en matériau végétal. Mais du fait de la rareté d'un tel matériau, des ustensiles en plastique sont de plus en plus utilisés. Les éleveurs considèrent cependant qu'ils ne permettent pas d'avoir un bon caillage car la buée descend dans le caillé et le rend moins ferme.

Différents types de calebasses sont utilisés au cours des différentes étapes de collecte et de transformation du lait (figure 9) pour préserver sa qualité hygiénique et assurer un bon caillage : le birdugal (calebasse pour la traite des vaches une par une), le larugal (calebasse pour contenir tout le lait trait), le kirdigal (calebasse pour garder le lait frais réservé pour le repas et les visiteurs), le penndirgal (calebasse pour cailler le lait issu de la traite), et le kuturngal (calebasse pour recueillir la matière grasse).

L'extraction du beurre se fait avec une baratte ou une gourde qui est un procédé plus rapide. En général, le beurre de vache (nebbam) est extrait par les femmes mais les hommes en transhumance

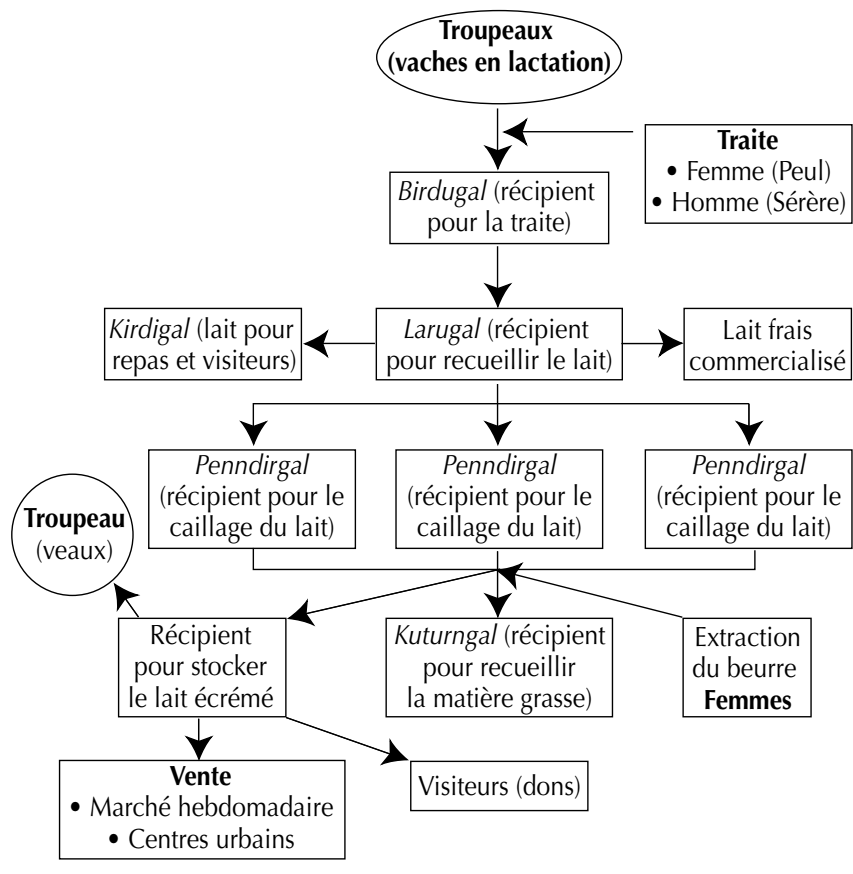

Figure 9 : destination de la production laitière traite des troupeaux de la zone sylvopastorale. 
peuvent être amenés à le faire. La quantité de lait frais nécessaire pour faire un litre de beurre est estimée à 25 à 30 litres (24).

Le lait des petits ruminants présente des avantages comparé à celui de la vache. Le caillé est comparable à celui du lait reconstitué à partir de la poudre de lait et il ne change pas à l'état frais si de l'eau y est rajoutée. Il est très énergétique et permet aussi à l'enfant d'éviter les diarrhées. Ce constat (densité du lait plus grande que celle de la vache et excellente valeur nutritionnelle, notamment pour les enfants) a été fait aussi par Waelti et coll. (29).

Le lait est apprécié selon sa teneur en matière grasse qui augmente progressivement d'environ $2,5 \mathrm{~g} / \mathrm{L} /$ semaine pour atteindre au moment du tarissement environ 80 à $90 \mathrm{~g} / \mathrm{L} / \mathrm{semaine}$, tandis que celle en lactose diminue après la saillie (18). Pour cette raison, le lait d'un animal adulte ou d'une vieille vache est considéré comme étant meilleur que celui d'une jeune femelle ; il en est de même du lait d'une vache gravide en lactation.

\section{Commercialisation des produits laitiers}

Parmi les revenus d'origine agricole, les produits laitiers occupent, dans la zone de forage de Thiel, la troisième place ( 8 p. 100) après la vente de bovins et de petits ruminants et avant les revenus tirés des productions agricoles (10). Le beurre reste en ZSP une source importante de revenus malgré la faible production $(3,2 \mathrm{~L} / \mathrm{mois} /$ ménage en saison des pluies et 0,2 L/mois/ménage en moyenne en saison sèche).

Les activités relatives à la commercialisation de lait frais sont menées par un nombre très réduit de familles. Cependant, à partir de la ville de Dahra, un dispositif de commercialisation du lait frais s'est mis en place en 1991. Les quantités collectées ont baissé du fait du retrait de la société Nestlé. Des organisations non gouvernementales et des privés ont pris le relais mais les quantités faisant l'objet de commercialisation sont encore très faibles.

La commercialisation du lait caillé écrémé est plus répandue. Elle a lieu notamment les jours de marché hebdomadaire. Chez les Peuls, ce sont les femmes qui s'en occupent, mais chez les Sérères en transhumance en ZSP, ce sont les hommes. Différents ustensiles sont utilisés lors de la vente (calebasses en bois ou en plastique et bidons). En 2001, le prix était environ de $200 \mathrm{Fcfa} / \mathrm{L}$ au niveau du marché hebdomadaire de Dahra.

\section{- CONCLUSION}

La production laitière en ZSP est en relation étroite avec la quantité d'eau que renferment les fourrages dans les pâturages naturels. Les mares qui sont le lieu d'abreuvement du cheptel constituent aussi un autre élément important dans la quantité de lait traite. Ainsi, dans la stratégie d'amélioration de la production laitière, la place de l'eau d'abreuvement doit être mieux prise en compte.

Les bovins continuent à fournir l'essentiel de la production laitière en ZSP. Cependant, les petits ruminants, notamment les chèvres, font de plus en plus l'objet de traite, ce qui contribue à augmenter les possibilités de traite, notamment en saison sèche.

L'utilisation de la complémentation alimentaire en saison sèche est pour l'instant limitée aux localités proches des centres urbains du fait des possibilités de vente du lait à des prix plus rémunérateurs. Le renforcement de la production laitière pendant cette période constitue un enjeu essentiel dans la bataille contre l'importation concurrente de produits laitiers dans de telles zones d'élevage.

Le beurre de vache constitue le produit le plus vendu. Le lait caillé fait l'objet d'une commercialisation de plus en plus importante sur les marchés hebdomadaires. La poursuite de la commercialisation $\mathrm{du}$ lait frais se fait difficilement sous l'impulsion d'organisations non gouvernementales et d'organisations de producteurs.

\section{Remerciements}

Les auteurs remercient M. Alioune Seck, responsable de la société Nestlé à Dahra, d'avoir bien voulu mettre à leur disposition les statistiques de la société. Ils remercient également MM. Gustave Gintzburger et Bernard Toutain du Cirad d'avoir accepté de relire le manuscrit.

\section{BIBLIOGRAPHIE}

1. AUBREVILLE A., 1949. Climats, forêts et désertification de l'Afrique tropicale. Paris, France, Société d'éditions géographiques et coloniales, 351 p.

2. BA C., 1989. Place du lait dans les systèmes pastoraux sahéliens ; exposés introductifs. In : Séminaire régional sur les systèmes de production du lait et de la viande au Sahel, Dakar, Sénégal, 22-26 nov. 1989, p. 24-31.

3. BARON J., 1955. Etude des procédés utilisés par les Maures pour empêcher les jeunes animaux de téter. Revue Elev. Méd. vét. Pays trop., $8: 15-23$.

4. BARRAL H., BENEFICE E., BOUDET G., DENIS J.P., DE WISPELAERE G., DIAITE I., DIAW O.T., DIEYE K., DOUTRE M.P., MEYER J.F., NOEL J., PARENT G., PIOT J., PLANCHENAULT., SANTOIR C., VALENTIN C., VALENZA J., VASSILIADES G., 1983. Systèmes de production d'élevage au Sénégal dans la région du Ferlo ; synthèses de fin d'étude d'une équipe de recherches pluridisciplinaires. Paris, France, Orstom, 172 p.

5. BERNUS E., 1984. L'homme et l'animal concurrents - Problèmes d'écologie pastorale sahélienne. In : Le développement rural en questions ; paysages, espaces ruraux, systèmes agraires; Maghreb, Afrique noire, Mélanésie. Paris, France, Orstom, p. 111-122. (Coll. Mémoires $n^{\circ} 106$ )

6. DENIS J.P., 1971. L'intervalle entre les vêlages chez le zébu Gobra (Peulh sénégalais). Revue Elev. Méd. vét. Pays trop., 24 : 635-647.

7. DENIS J.P., 1978. Note sur le sex-ratio chez le zébu Gobra au CRZ de Dahra. Revue Elev. Méd. vét. Pays trop., 31 : 443-445.

8. DENIS J.P., THIONGANE A.I., 1974. Analyse de la lactation de vaches Pakistanaises au Sénégal. Revue Elev. Méd. vét. Pays trop., 27 : 331-346.

9. DENIS J.P., VALENZA J., 1971. Extériorisation des potentialités génétiques du zébu peulh sénégalais. Revue Elev. Méd. vét. Pays trop., 24 : 409-418.

10. DIA T., 1997. Etude des revenus dans les unités pastorales du PAPEL : le cas de Thiel. Document de travail. Dahra, Sénégal, ISRA/CRZ, 2 p.

11. DIOUF A., LAMBLIN E.F., 2001. Monitoring land-cover changes in semi-arid regions; remote sensing data and field observations in the Ferlo, Sénégal. J. arid Environ., 48: 129-148.

12. FAYOLLE A.F., COSTIOU P., GRANGE M., 1974. Valorisation du cheptel bovin zone sylvopastorale de la République du Sénégal - Rapport d'enquêtes. Dakar Hann, Sénégal, Lnerv, p. 126 p.

13. GASTON A., DIEYE H., BERGES J.C., CITEAU J., 1987. Evaluation de la productivité primaire herbacée annuelle au Sahel sénégalais. Saison des pluies 1986. Veille climat., $17: 49-54$.

14. GUERIN H., 1987. Alimentation des ruminants domestiques sur pâturages naturels sahéliens et sahélo-soudaniens : étude méthodologique dans la région du Ferlo au Sénégal. Thèse Doct. Ing., ENSA, Montpellier, France, $284 \mathrm{p}$.

15. KING J., 1989. Influence du climat et de I'alimentation sur les besoins en eau du bétail en Afrique tropicale. Addis-Abéba, Ethiopie, Cipea, 103 p. (Rapport de recherche $\mathrm{n}^{\circ}$ )

16. LABOUCHE C., 1957. La physiologie de la lactation en milieu tropical. II. Influence de la saison sur la vitesse de chute de la production laitière (observations recueillies en zone subguinéenne de la presqu'île du Cap vert). Revue Elev. Méd. vét. Pays trop., 10 : 249-256.

17. LABOUCHE C., 1957. La physiologie de la lactation en milieu tropical. I. Etude des courbes de lactation recueillies en zone subguinéenne de la presqu'île du Cap vert (Sénégal). Revue Elev. Méd. vét. Pays trop., $10: 27-39$. 
18. LABOUCHE C., PEYTAVIN A., 1957. Sur la composition chimique des laits tropicaux : influence du stade de la lactation sur les teneurs en graisse, lactose, calcium et phosphore. Revue Elev. Méd. vét. Pays trop., $10: 373-382$.

19. LAKE L.A., NDOUR N., THIOBANE M., BA A., 2003. Evolution of vegetal production in Sahelian natural range lands: the case of North West Senegal in the 1990s. Sécheresse, 14: 5-13.

20. MEYER C., DUTEURTRE G., 1998. Equivalents lait et rendements en produits laitiers : modes de calculs et utilisation. Revue Elev. Méd. vét. Pays trop., $51: 247-257$.

21. MINISTERE DE L'AGRICULTURE ET DE L'ELEVAGE, 2000. Recensement national de l'Agriculture - 1998/99. Rapport général du recensement de l'agriculture pluviale, vol. 4. Dakar, Sénégal, ministère de l'Agriculture et de l'Elevage, $536 \mathrm{p}$.

22. POUILLON F., 1984. Evaluation de l'élevage bovin dans la zone sahélienne du Sénégal. Etude sociologique. Paris, France, ministère de la Coopération, p. 69-112.

23. PREVOST Y., 1990. Analyse spatiale de la pression animale comme facteur de désertification dans le nord du Sénégal. Télédétection et sécheresse. Paris, France, John Libbey Eurotext, p. 241-251.

24. SANTOIR C., 1982. Contribution à l'exploitation du cheptel. Région du Ferlo (Sénégal). Dakar, Sénégal, Orstom, 48 p.
25. SCHAFFER J., 1994. Economie pastorale dans le Ferlo des forages ; Bouteyni et Widou Thiengoly en 1994. Rapport de recherche PAPF. Saint Louis, Sénégal, GTZ, 67 p.

26. SIMONNET H., 1955. L'eau : besoins de l'organisme, métabolisme, influence de l'abreuvement sur la production animale. Revue Elev. Méd. vét. Pays trop., 8 : 179-208.

27. STANCIOFF A., STALJANSSENS M., TAPPAN G., 1986. Cartographie et télédétection des ressources de la République du Sénégal : étude de la géologie, de I'hydrologie, des sols, de la végétation et des potentiels d'utilisation. Brookings, SD, USA, Remote Sensing Institute, South Dakota State University, $653 \mathrm{p}$.

28. VALENZA J., DIALLO A.K., 1972. Etude des pâturages naturels du Nord Sénégal. Etudes agrostologiques. Maisons-Alfort, France, lemvt, $311 \mathrm{p}$.

29. WAELTI P., KONE I., BARRY A., DIARRA M., NIANGADO O., 2004. Production laitière des petits ruminants, lutte contre la malnutrition et diversification des revenus dans la commune de Cinzana (Mali). Etud. Rech. sahél., 8-9 : 117-125.

Accepté le 15.02.2010

\section{Summary}

Diop A.T., Ickowicz A., Diène M., Nzimulinda J.C. Milk Production in the Sylvopastoral Zone of Senegal: Variation Factors and Local Populations' Management System

In the Sahelian region of Senegal, milk production in pastoral systems is subjected to inter- and intra-annual variations because the animal feed is almost exclusively based on natural resources. Studies conducted from a monitoring set-up in pastoralist settlements and in station, and data from Nestle Company have shown that milk production was highly related to the milk collection date, but with a low correlation with the total amount of rainfall. The relationship between total milk production and the normalized difference vegetation index (NDVI) was polynomial. The production peak was reached when the water content in forage was $70 \%$. Surveys on pastoralists (men and women) belonging to different ethnic groups and living in different production subsystems (Walo, Djoloff and Ferlo) showed that the duration of lactation, number of cows and animal species were the main factors of variation of milk production. The actors in charge of milk production management differed depending on the ethnic group. Milk curdling and butter making were the two preserving methods used, and five types of gourds were used in the process. Dairy products still held a major place in farmers' incomes and skim milk curd was the most marketed product.

Keywords: Milk production - Biomass - Pasture - Moisture content - Remote sensing - Senegal.

\section{Resumen}

Diop A.T., Ickowicz A., Diène M., Nzimulinda J.C. Producción lechera en la zona silvo-pastoril de Senegal: estudio de los factores de variación y de los modos de gestión de las poblaciones locales

En la región sahelina de Senegal, la producción lechera de los sistemas pastoriles conoce variaciones inter anuales e intra anuales, debido al hecho de que la alimentación del hato está basada casi exclusivamente en los recursos naturales. Los estudios llevados a cabo a partir de un dispositivo de seguimiento en los campamentos de criadores y en estación y los datos de la sociedad Nestlé han mostrado que la producción lechera está fuertemente relacionada con la fecha de inicio de la colecta, y más débilmente a la pluviosidad total. La relación entre la producción lechera total y los índices de vegetación normalizados (NDVI) fueron de tipo polinomio. El pico de la producción se alcanzó en el momento en donde el contenido de agua en los forrajes fue de $70 \%$. Encuestas llevadas a cabo con los pastores (hombres y mujeres), pertenecientes a diferentes etnias y residentes en los diferentes sistemas de sub producción (Walo, Djoloff y Ferlo), indicaron que la duración de la lactación, el número de vacas y las especies animales constituyeron los principales factores de variación de la producción lechera. En función de la etnia a la cual pertenecían, diferentes actores fueron responsables de la gestión de la leche. La cuajada y la extracción de la mantequilla constituyeron los dos modos de conservación y cinco tipos de calabazas fueron utilizadas durante el proceso. Los productos lecheros ocuparon aún así un lugar importante en los ingresos agrícolas y la comercialización de la leche cortada descremada fue la más común.

Palabras clave: Producción lechera - Biomasa - Pastizal Contenido de humedad - Teledetección - Senegal. 